\title{
ASYMPTOTIC PATHS FOR SUBSOLUTIONS OF QUASILINEAR ELLIPTIC EQUATIONS
}

\author{
JuHA HEINONEN
}

Let $u$ be an entire lower semicontinuous subsolution to the quasilinear elliptic equation $\operatorname{div} A(x, \nabla u)=0$ in $\mathbb{R}^{n}$. It is shown that if $u$ is not bounded above, then there exists a path going to infinity along which $u$ tends to infinity. The result extends works of Talpur, Fuglede, and others. Growth aspects of subsolutions are also studied.

\section{INTRODUCTION}

A classical result of $F$. Iversen [13] states that if $f$ is a non-constant entire analytic function, there is a continuous path $\Gamma$ in the complex plane going to infinity such that $f(z) \rightarrow \infty$ as $z \rightarrow \infty$ along $\Gamma$. Such a path is called an asymptotic path. Today a relatively simple proof of that result can be given which applies to all continuous subharmonic functions in $\mathbb{R}^{2}$ (thus replacing $\log |f|$ in Iversen's theorem), see [7]. To prove the result for general subharmonic functions in $\mathbb{R}^{n}, n \geq 2$, is a considerably more difficult task. M. N. M. Talpur [19] was the first to show that in the plane an asymptotic path can always be found; shortly after B. Fuglede [4] settled the problem in every dimension by using Brownian paths and fine potential theory. Since then several different proofs for Fuglede's theorem have appeared including a probabilistic proof (T. Lyons [14]), another proof in terms of fine potential theory (B. Fuglede [5]), and at least two "classical" proofs (L. Carleson [2] and W. K. Hayman [8]). See also B. Davis and J. L. Lewis [3].

This paper was written while I was in Bonn in the fall 1987. I take this opportunity to thank the Mathematics Department in Bonn for its hospitality. I also thank Tero Kilpeläinen who read the manuscript and shortened some of my arguments. 
In this paper we prove the existence of an asymptotic path for subsolutions of the quasilinear elliptic equation

$$
\operatorname{div} A(x, \nabla u)=0
$$

where $A(x, \nabla u) \cdot \nabla u \approx|\nabla u|^{p}$ and $1<p<\infty$. The accurate description of (1.1) is given in Section 2.

We naturally have to explain precisely what we mean by a subsolution in this connection. Indeed, we consider so-called $A$-subharmonic functions which were introduced in [10]. Let us recall the definition. A continuous (weak) solution to the equation (1.1) is called $A$-harmonic, and a function $u$ defined in an open set $\Omega \subset \mathbb{R}^{n}, n \geq 2$, is called $A$ subharmonic if

(i) $u$ is upper semicontinuous (u.s.c.),

(ii) $-\infty \leq u<\infty$, and

(iii) for each domain $D \subset \subset \Omega$ and each $A$-harmonic $h \in C(\bar{D}), u \leq h$ in $\partial D$ implies $u \leq h$ in $D$.

Our first main theorem now reads as follows:

1.2. Theorem. Suppose that $u$ is $A$-subharmonic in $\mathbb{R}^{n}$ and not bounded above. There exists a continuous path $\Gamma$ going to infinity such that $u(x) \rightarrow \infty$ as $x \rightarrow \infty$ along $\Gamma$.

It was shown by $T$. Kilpeläinen and the author in [10] that each subsolution to (1.1) has a unique $A$-subharmonic representative so that Theorem 1.2 is about subsolutions as well. On the other hand, $A$-subharmonic functions are not subsolutions in general (see Section 2).

In order to appreciate the existence of an asymptotic path, it is perhaps worth mentioning that, if $1<p \leq n$, for every operator $A$ there are entire $A$-subharmonic functions (in fact, entire subsolutions to (1.1)) which are non-negative, unbounded, and vanish at a dense set of points in $\mathbb{R}^{n}$. In the subharmonic case $A(x, h)=h$ this is classical and well known, and the general case is treated in [11].

Another classical result of complex analysis states that if $f$ is an entire function having $k$ distinct asymptotic values, $k \geq 2$, then the lower order of $f$ is at least $\frac{1}{2} k$. This is the famous and seminal Denjoy-Ahlfors theorem, see e.g. [1]. For a subharmonic function $u$ in $\mathbb{R}^{n}$ the analogous problem asks the relationship between the lower order of $u$ and the number $k \geq 2$ of distinct components of the set $\{u \geq L\}$ for some large number $L$. Talpur [19], [20] and Hayman [8] proved that the lower order is at least a positive constant depending only on $n$ and $k$, and this constant tends to infinity with $k$. The second main result of this paper, Theorem 4.7 in Section 4, is an extension of that theorem to $A$ - 


\section{HEINONEN}

subharmonic functions. We thus extend the work of V. M. Miklyukov [17] who proved Theorem 4.7 for continuous subsolutions of (1.1).

Our proofs are based on ideas taken from [8] and [3] as well as on some techniques and results from nonlinear potential theory [10], [12]. The critical step is to establish the existence of an asymptotic continuum. The existence of an actual path can then be deduced as in [8]. The main novelty is that the very properties of subharmonic functions (submeanvalue property, representation by potentials, linear structure) play no rôle in our reasoning. On the other hand, we do not know whether the path in Theorem 1.2 can be chosen to be sectionally polygonal as it is the case for ordinary subharmonic functions [2], [14], [5]. Neither do we know whether there are reasonable quantitative estimates for the length of the path, cf. [3]. What is more, it would be extremely interesting to know whether there is a probabilistic approach, or an approach similar to that of Fuglede [4], [5], to the questions of this paper.

There is an analogy between potential theory and function theory in higher dimensions as well. Namely, if $f: \mathbb{R}^{n} \rightarrow \mathbb{R}^{n}$ is a quasiregular mapping, then $\log |f|$ is an $A$-subharmonic function for an appropriate operator $A, A(x, h) \cdot h \approx|h|^{n}$, see e.g. [18], [6]. Thus our results apply to entire quasiregular mappings and can be viewed as extensions of the results of Iversen and Ahlfors. We notice however that in this particular case different methods can be used; indeed, Iversen's theorem for quasiregular mappings was proved by $O$. Martio et al. in $[\mathbf{1 5}, 3.18]$ and a generalization of Ahlfors's theorem is due to V. M. Miklyukov [17].

Our notation is standard. Throughout, $\Omega$ is a connected open set in $\mathbb{R}^{n}, n \geq 2$, and $D \subset \subset \Omega$ means that $\bar{D}$, the closure of $D$, is compact in $\Omega$. If $B=B(x, r)=\left\{y \in \mathbb{R}^{n}:|x-y|<r\right\}$ is an open $n$-ball and $\sigma>0$, then $\sigma B=B(x, \sigma r)$, and similarly for the closed ball $\sigma \bar{B}=$ $\bar{B}(x, \sigma r)$. The Lebesgue $n$-measure of a measurable set $E$ is $|E|$, and the integral averages are marked as usual with $\frac{1}{|E|} \int_{E} u d x=f_{E} u d x$. We let $c, c_{0}, c_{1}, \ldots$ denote various positive constants which are not necessarily the same at each occurrence, and $c(a, b, \ldots)$ denotes a constant depending on $a, b, \ldots$.

\section{Preliminaries}

In this section we recall the definition and some basic properties of $A$-subharmonic functions.

Let $A: \mathbb{R}^{n} \times \mathbb{R}^{n} \rightarrow \mathbb{R}^{n}$ be an operator satisfying the following assumptions for some numbers $1<p<\infty$ and $0<\alpha \leq \beta<\infty$ :

the function $x \mapsto A(x, h)$ is measurable for all $h \in \mathbb{R}^{n}$ and the function $h \mapsto A(x, h)$ is continuous for a.e. $x \in \mathbb{R}^{n}$; 
for all $h \in \mathbb{R}^{n}$ and a.e. $x \in \mathbb{R}^{n}$

$$
\begin{gathered}
A(x, h) \cdot h \geq \alpha|h|^{p}, \\
|A(x, h)| \leq \beta|h|^{p-1}, \\
\left(A\left(x, h_{1}\right)-A\left(x, h_{2}\right)\right) \cdot\left(h_{1}-h_{2}\right)>0
\end{gathered}
$$

whenever $h_{1} \neq h_{2}$, and

$$
A(x, \lambda h)=|\lambda|^{p-2} \lambda A(x, h)
$$

for all $\lambda \in \mathbb{R}, \lambda \neq 0$.

A real valued function $u$ in $\Omega$ is said to be $A$-harmonic if it is a continuous weak solution to the equation

$$
\operatorname{div} A(x, \nabla u)=0 .
$$

In other words, $u$ is $A$-harmonic if it is continuous, locally in the Soboley space $W_{p}^{1}(\Omega)$, and satisfies

$$
\int_{\Omega} A(x, \nabla u) \cdot \nabla \varphi d x=0
$$

for all $\varphi \in C_{0}^{\infty}(\Omega)$. It is well known that weak solutions to (2.6) are actually continuous.

A function $u$ in $\Omega,-\infty \leq u<\infty$, is $A$-subharmonic if it is upper semicontinuous (u.s.c.) and satisfies the comparison principle: for each domain $D \subset \subset \Omega$ and each $A$-harmonic function $h \in C(\bar{D}), u \leq h$ in $\partial D$ implies $u \leq h$ in $D$. The function $u$ is $A$-superharmonic if and only if $-u$ is $A$-subharmonic.

Unfortunately, the sum of two $A$-subharmonic functions is not $A$ subharmonic in general. To some extent this lack of linearity can be compensated by using the following simple observations: if $u$ is $A$ subharmonic, so is $\lambda u+\mu$ whenever $\lambda \geq 0$ and $\mu \in \mathbb{R}$; if $u$ and $v$ are $A$-subharmonic, so is $\max (u, v)$.

The model equation to (2.6) is the so-called $p$-Laplace equation

$$
\operatorname{div}\left(|\nabla u|^{p-2} \nabla u\right)=0,
$$

and in this case the terminology $p$-harmonic and $p$-subharmonic is customary. The fundamental $p$-subharmonic function in $\mathbb{R}^{n}$ is

$$
u(x)= \begin{cases}-|x|^{\frac{p-n}{p-1}}, & p \neq n, \\ \log |x|, & p=n .\end{cases}
$$




\section{HEINONEN}

A subsolution to the equation (2.6) is a function $u$ in $\operatorname{loc} W_{p}^{1}(\Omega)$ satisfying

$$
\int_{\Omega} A(x, \nabla u) \cdot \nabla \varphi d x \leq 0
$$

for all non-negative $\varphi \in C_{0}^{\infty}(\Omega)$. It was shown in [10] that if $u$ is a subsolution to (2.6), then $u$ has a unique $A$-subharmonic representative defined by

$$
u(x)=\underset{y \rightarrow x}{\operatorname{ess} \lim \sup } u(y) .
$$

On the other hand, if $1<p \leq n$, then an $A$-subharmonic function need not be locally in $W_{p}^{1}$ as displayed by the examples in (2.7). If $p>n$, all $A$-subharmonic functions are continuous and thus subsolutions, see $[10,3.20]$; in fact, for $p>n$ the considerations in this paper could be somewhat simpler.

2.9. Proposition. [10] If $u$ is $A$-subharmonic in $\Omega$, then (2.8) holds at each point $x \in \Omega$. Further, for each domain $D \subset \subset \Omega$ there is a decreasing sequence of $A$-subharmonic functions $u_{j} \in C(\bar{D})$ such that $\lim u_{j}=u$ in $D$; the functions $u_{j}$ are subsolutions of (2.6) in $D$.

Both the "ess lim sup" property and the approximation property will be used in this paper. We do not explicitly need the fact that the approximating functions are subsolutions. Observe that if $u_{j}$ is any decreasing sequence of $A$-subharmonic functions, then $u=\lim u_{j}$ is $A$ subharmonic (possibly $\equiv-\infty$ ). It is also true that being $A$-subharmonic is a local property [10].

We shall make use of the following weak submean-value inequality which is by now rather standard for subsolutions to equations of the type (2.6).

2.10. Proposition. Suppose that $u$ is non-negative and $A$-subharmonic in $\Omega$. If $B=B\left(x_{0}, r\right) \subset \Omega$ and $0<\sigma<1$, then

$$
\sup _{B\left(x_{0}, \sigma r\right)} u \leq c_{0}(1-\sigma)^{-\xi} f_{B\left(x_{0}, r\right)} u d x
$$

where $\xi=n$, if $p<n, \xi=2 p$, if $p \geq n$, and $c_{0}=c_{0}(n, p, \alpha / \beta)>0$.

Proof: Since $u$ is lower bounded, it is a subsolution of (2.6) by Corollary 3.13 in [10]. Thus (2.11) follows from the estimate [10, (2.19)] together with the fact that $\operatorname{ess} \sup u=\sup u$. 
2.12. Comparison principle. $[10,3.7]$. Suppose that $\Omega$ is bounded and $u,-v$ are $A$-subharmonic in $\Omega$. If

$$
\limsup _{y \rightarrow x} u(y) \leq \liminf _{y \rightarrow x} v(y)
$$

for each $x$ in $\partial \Omega$ and if the left hand side and the right hand side are not simultaneously $+\infty$ or $-\infty$, then $u \leq v$ in $\Omega$.

\section{ON THE COMPONENTS OF $\{u \geq \varepsilon\}$}

The goal of this section is to prove

3.1. Proposition. Suppose that $u$ is $A$-subharmonic in a ball $B=$ $B\left(x_{0}, r\right)$ and $0 \leq u<1$. Suppose that $x_{1}, x_{2}$ are two points in $\frac{1}{2} B$ such that $u\left(x_{1}\right), u\left(x_{2}\right) \geq 2 \varepsilon>0$. There is a number $\delta_{0}=\delta_{0}(n, p, \varepsilon, \alpha, \beta)>0$ such that if $\left|x_{1}-x_{2}\right|<\delta_{0} r$, then $x_{1}$ and $x_{2}$ belong to the same component of the compact set $\{u \geq \varepsilon\} \cap \frac{3}{4} \bar{B}$.

The Proposition 3.1 is the main ingredient for the proof of Theorem 1.2. In fact, once we have established Proposition 3.1 and the existence of an asymptotic continuum, we may invoke the argument of Hayman [8, pp. 188-191] and deduce that the required path actually exists.

We start with a lemma, similar to Theorem 4.11 in [8].

3.2. Lemma. Suppose that $u$ is $A$-subharmonic in $\Omega$ and that $D \subset \subset \Omega$ is a domain. Let $D_{L}=\{x \in \Omega: u(x) \geq L>-\infty\} \cap \bar{D}$ and let $K(L)$ be a component of $D_{L}$. Set

$$
v(x)= \begin{cases}u(x), & x \in K(L), \\ L, & x \in D \backslash K(L) .\end{cases}
$$

Then $v$ is $A$-subharmonic in $D$.

Proof: Suppose first that $u$ is continuous in a neighborhood of $\bar{D}$. Then $v$ is continuous in $D$. Let $U \subset \subset D$ be a domain and let $h \in C(\bar{U})$ be $A$-harmonic in $U$ with $v \leq h$ in $\partial U$. If the open set $V=\{x \in$ $U: h(x)<v(x)\}$ is not empty, then necessarily $v=u$ in $V$. Thus for $y \in \partial V$ we have $\lim _{x \rightarrow y, x \in V} u(x)=\lim _{x \rightarrow y, x \in V} v(x)=h(y)$, whence $v=u \leq h<v$ in $V$, which is absurd. It follows that $v$ is $A$-subharmonic if $u$ is continuous.

To prove the general case, we pick a decreasing sequence of functions $u_{j}$ such that $u_{j} \rightarrow u$ in $\bar{D}$ and each $u_{j}$ is $A$-subharmonic in a fixed neighborhood of $\bar{D}$, see Proposition 2.9. Fix a point $x_{0} \in K(L)$ and let 


\section{HEINONEN}

$K_{j}(L)$ be the $x_{0}$-component of the compact set $\left\{u_{j} \geq L\right\} \cap \bar{D}$. Then $K_{j}(L) \supset K_{j+1}(L)$. Setting

$$
v_{j}(x)= \begin{cases}u_{j}(x), & x \in K_{j}(L), \\ L, & x \in D \backslash K_{j}(L),\end{cases}
$$

we obtain a decreasing sequence of $A$-subharmonic functions converging to an $A$-subharmonic function $v_{0}$ in $D$. We show that $v_{0}=v$ in $D$. Indeed, it can be easily inferred that $K(L)=\cap_{j} K_{j}(L)$, whence for $x \in K(L)$ we have $v_{0}(x)=\lim v_{j}(x)=\lim u_{j}(x)=u(x)$. If, on the other hand, $x \in D \backslash K(L)$, then $x$ is not in $K_{j}(L)$ for $j$ big enough, whence $v_{0}(x)=L=v(x)$. Thus $v_{0}=v$ is $A$-subharmonic in $D$ as required. The lemma is proved.

Next we recall the definition for the variational $p$-capacity. If $F$ is a compact subset of a ball $B$, the $p$-capacity of the pair $(F, B)$ is the number

$$
\operatorname{cap}_{p}(F, B)=\inf _{u} \int_{B}|\nabla u|^{p} d x
$$

where $u$ runs through the set $\left\{u \in C_{0}^{\infty}(B): u \geq 1\right.$ on $\left.F\right\}$. If $U \subset B$ is open, we set

$$
\operatorname{cap}_{p}(U, B)=\sup _{F \subset U \text { compact }} \operatorname{cap}_{p}(F, B)
$$

and, finally, for an arbitrary $E \subset B$

$$
\operatorname{cap}_{p}(E, B)=\inf _{U \supset E \text { open }} \operatorname{cap}_{p}(U, B)
$$

The number $\operatorname{cap}_{p}(E, B)$ is the variational $p$-capacity of the pair $(E, B)$. It is standard that for a Borel set $E \subset B$

$$
\operatorname{cap}_{p}(E, B)=\sup _{F \subset E \text { compact }} \operatorname{cap}_{p}(F, B) .
$$

If $E$ is a subset of a ball $B$, then one denotes by $\hat{R}_{E}^{1}(B ; A)$ the $A$-potential of $E$ in $B$. The function $\hat{R}_{E}^{1}=\hat{R}_{E}^{1}(B ; A)$ is the lower regularization of the réduite $R_{E}^{1}=\inf \{u: u$ is non-negative and $A$-superharmonic in $B$ with $u \geq 1$ on $E$ \},

$$
\hat{R}_{E}^{1}(x)=\liminf _{y \rightarrow x} R_{E}^{1}(y) .
$$

Basically classical arguments can be used to show that the function $\hat{R}_{E}^{1}$ is $A$-superharmonic in $B$; see [11] for this. 


\section{HEINONEN}

The following lemma, which is essentially due to V. G. Maz'ya [16], is Lemma 5.2 in [9] (in [9] it was assumed that $E$ is compact and $1<p \leq n$ but the general case is similar).

3.3. LemmA. Let $E$ be a Borel set in $\sigma \bar{B}=\bar{B}\left(x_{0}, \sigma r\right), 0<\sigma<1$, and let $u=\hat{R}_{E}^{1}(B ; A)$ be the $A$-potential of $E$ in $B$. Then

$$
u(x) \geq \operatorname{cr}^{\frac{p-n}{p-1}} \operatorname{cap}_{p}(E, B)^{\frac{1}{p-1}}
$$

for all $x \in \sigma \bar{B}$. The constant $c$ depends only on $n, p, \alpha, \beta$, and $\sigma$.

By using Lemma 3.3 we derive a Wiener-type estimate for $A$-subharmonic functions, cf. [3, Lemma 1], [16]. For brevity, we write

$$
\varphi_{p}(z, E, s)=\operatorname{cap}_{p}(E \cap B(z, s), B(z, 2 s)) s^{p-n} .
$$

3.4. Lemma. Suppose that $u$ is $A$-subharmonic in $B=B\left(x_{0}, r\right)$ and $0 \leq u<1$. Let $U=\left\{x \in B: u(x)<\frac{1}{2} u\left(x_{0}\right)\right\}$. Then

$$
u\left(x_{0}\right) \leq 2 \exp \left(-c_{1} \int_{0}^{r / 2} \varphi_{p}\left(x_{0}, U, s\right)^{\frac{1}{p-1}} \frac{d s}{s}\right)
$$

where $c_{1}=c_{1}(n, p, \alpha, \beta)>0$.

Proof: Write $u_{1}=\max \left(u-\frac{1}{2} u\left(x_{0}\right), 0\right)$. Then $E=\left\{u_{1}=0\right\} \supset U$, and we may clearly assume that $E \neq \emptyset$. If we denote $a_{k}=\varphi_{p}\left(x_{0}, E, 2^{-k} r\right)$, $k=1,2, \ldots$, it is not difficult to show that

$$
\sum_{k=1}^{\infty} a_{k}^{\frac{1}{p^{-1}}} \geq c \int_{0}^{r / 2} \varphi_{p}\left(x_{0}, E, s\right)^{\frac{1}{p-1}} \frac{d s}{s}
$$

where $c>0$ depends only on $n$ and $p$. Thus it suffices to verify that

$$
u_{1}\left(x_{0}\right) \leq \exp \left(-c_{1} \sum_{k=1}^{\infty} a^{\frac{1}{k^{-1}}}\right) .
$$

To do this, let $v_{1}=\hat{R}_{E_{1}}^{1}(B ; A)$ be the $A$-potential of $E_{1}=E \cap \frac{1}{2} B$ in $B$. By Lemma 3.3

$$
\min _{\frac{1}{2} B} v_{1} \geq c a \frac{1}{p^{-1}} \geq 1-e^{-c a \frac{1}{p^{-1}}}
$$

and since $1-u_{1} \geq v_{1}$, we obtain

$$
\max _{\frac{1}{2} B} u_{1} \leq e^{-c a} p^{\frac{1}{p^{-1}}}
$$




\section{HEINONEN}

where $c=c(n, p, \alpha, \beta)>0$. Now the function $u_{2}=e^{c a p^{\frac{1}{p-1}}} u_{1}$ is $A$ subharmonic with $0 \leq u_{2}<1$ in $\frac{1}{2} B$ and we similarly obtain

$$
\max _{\frac{1}{4} B} u_{2} \leq e^{-c a 2^{\frac{1}{p^{-1}}}}
$$

whence

$$
\max _{\frac{1}{4} B} u_{1} \leq e^{-c\left(a \bar{p}^{\frac{1}{1}}+a_{2}^{\frac{1}{p-1}}\right)} .
$$

Continuing this way we arrive at the estimate

$$
\max _{2-k B} u_{1} \leq e^{-c \sum_{j=1}^{k} a_{j}^{\frac{1}{p-1}}}
$$

and letting $k \rightarrow \infty$ yields (3.6). The lemma is thereby proved.

Proof of Proposition 3.1: To start with, we state the following simple consequence of the Poincare inequality: if $E$ is a Borel set in $R^{n}$, $x \in \mathbb{R}^{n}$, and $s>0$, then

$$
\theta(x, E, s) \leq c \varphi_{p}(x, E, s)
$$

where

$$
\theta(x, E, s)=\frac{|E \cap B(x, s)|}{|B(x, s)|}
$$

and $c=c(n, p)>0$. Indeed, it clearly suffices to prove (3.7) for compact sets $E$ and in that case the Poincare inequality implies for $\varphi \in C_{0}^{\infty}(B(x, 2 s)), \varphi \geq 1$ on $E \cap \bar{B}(x, s)$,

$$
|E \cap B(x, s)| \leq \int_{B(x, 2 s)}|\varphi|^{p} d x \leq c s^{p} \int_{B(x, 2 s)}|\nabla \varphi|^{p} d x .
$$

The estimate (3.7) is now immediate.

We proceed to prove the assertion in Proposition 3.1. For $i=1,2$ let $K_{i}$ be the $x_{i}$-component of the set $\{u \geq \varepsilon\} \cap \frac{3}{4} \bar{B}$. We assume that $K_{1} \cap K_{2}=\emptyset$ and derive a lower bound for $\left|x_{1}-x_{2}\right|$.

Set

$$
v_{1}(x)= \begin{cases}u(x), & x \in K_{1}, \\ \varepsilon, & x \in \frac{3}{4} B \backslash K_{1},\end{cases}
$$

and $u_{1}=v_{1}-\varepsilon$. Then $u_{1}$ is $A$-subharmonic in $\frac{3}{4} B$ by Lemma 3.2. Since $0 \leq u_{1}<1$ and $u_{1}\left(x_{1}\right) \geq \varepsilon$, Lemma 3.4 implies

$$
\varepsilon \leq 2 \exp \left(-c_{1} \int_{0}^{r / 8} \varphi_{p}\left(x_{1}, E_{1}, s\right)^{\frac{1}{p-1}} \frac{d s}{s}\right)
$$




\section{HEINONEN}

where $E_{1}=\frac{3}{4} B \backslash K_{1} \subset\left\{x \in \frac{3}{4} B: u_{1}(x)<\frac{1}{2} u_{1}\left(x_{1}\right)\right\}$. Suppose that $\theta\left(x_{1}, E_{1}, s\right) \geq c_{2}>0$ for $0<s_{2} \leq s \leq s_{1} \leq r / 8$. Then by (3.7) and (3.8)

or

$$
\varepsilon \leq 2 \exp \left(-c c_{2}^{\frac{1}{p^{-1}}} \int_{s_{2}}^{s_{1}} \frac{d s}{s}\right)=2 \exp \left(-c c_{2}^{\frac{1}{p^{-1}}} \log \frac{s_{1}}{s_{2}}\right)
$$

$$
s_{1} \leq c_{3} s_{2}
$$

where $c_{3}=(2 / \varepsilon)^{c c_{2}^{\frac{1}{1-p}}}, c=c(n, p, \alpha, \beta)>0$. By induction one easily infers that there is a decreasing sequence of numbers $r / 8=s_{0}>s_{1}>$ $s_{2}>\ldots>0$ converging to zero such that for $j=0,1,2, \ldots$

$$
2 s_{j+1} \leq s_{j} \leq c_{4} s_{j+1}, \quad c_{4}=2(2 / \varepsilon)^{c c_{2}^{\frac{1}{1-p}}}, \quad c=c(n, p, \alpha, \beta)>0
$$

and

$$
\theta\left(x_{1}, E_{1}, s_{j+1}\right)<c_{2} .
$$

Similarly as above we set

$$
v_{2}(x)= \begin{cases}u(x), & x \in K_{2}, \\ \varepsilon, & x \in \frac{3}{4} B \backslash K_{2},\end{cases}
$$

and $u_{2}=v_{2}-\varepsilon$. Then $u_{2}$ is $A$-subharmonic in $\frac{3}{4} B$ and $\left\{u_{2}>0\right\} \subset$ $K_{2} \subset E_{1}$. We invoke the inequality (2.11) and deduce from (3.9) and (3.10) that

$$
\begin{aligned}
\sup _{B\left(x_{1}, s_{j+1}\right)} u_{2} & \leq c_{0} 2^{\xi} f_{B\left(x_{1}, s_{j}\right)} u_{2} d x \\
& \leq c_{0} 2^{\xi} \frac{\left|\left\{u_{2}>0\right\} \cap B\left(x_{1}, s_{j}\right)\right|}{\left|B\left(x_{1}, s_{j}\right)\right|} \sup _{B\left(x_{1}, s_{j}\right)} u_{2} \\
& \leq c_{0} 2^{\xi} c_{2} \sup _{B\left(x_{1}, s_{j}\right)} u_{2} \\
& \leq \ldots \leq c_{0}^{j} 2^{j \xi} c_{2}^{j} \sup _{B\left(x_{1}, r / 8\right)} u_{2} .
\end{aligned}
$$

We now choose $c_{2}=c_{2}(n, p, \varepsilon, \alpha, \beta)>0$ such that $c_{0} 2^{\xi} c_{2}=\frac{1}{2}$. It follows that

$$
\sup _{B\left(x_{1}, s_{j+1}\right)} u_{2} \leq 2^{-j} \sup _{B\left(x_{1}, r / 8\right)} u_{2} \leq 2^{-j} .
$$

If $\left|x_{1}-x_{2}\right| \geq s_{2}$, the proof is finished since $s_{2} \geq c_{4}^{-2} s_{0}=\delta_{0}(n, p, \varepsilon, \alpha, \beta) r>$ 0 by (3.9). We may thus suppose that $\left|x_{1}-x_{2}\right|<s_{2}$. Let $j_{0}$ be the integer such that $s_{j_{0}+2} \leq\left|x_{1}-x_{2}\right|<s_{j_{0}+1}$. Then

$$
\varepsilon \leq \sup _{B\left(x_{1}, s_{j_{0}+1}\right)} u_{2} \leq 2^{-j_{0}}
$$

or $j_{0} \leq \log \frac{1}{\varepsilon} / \log 2$. It again follows that $\left|x_{1}-x_{2}\right| \geq s_{j_{0}+2} \geq c_{4}^{-j_{0}-2} s_{0}=$ $\delta_{0}(n, p, \varepsilon, \alpha, \beta) r>0$, and the proof of Proposition 3.1 is complete. 


\section{HEINONEN}

We close this section by stating a suitable corollary to Proposition 3.1.

3.11. Lemma. Suppose that $u$ is $A$-subharmonic in a ball $B=B\left(x_{0}, r\right)$ such that $u\left(x_{0}\right) \geq L+\varepsilon>-\infty$ and $\sup _{B} u \leq L+M<\infty$, where $\varepsilon$ and $M$ are positive constants. There is $\delta_{0}=\delta_{0}(n, p, \alpha, \beta, \varepsilon, M)>0$ such that if $\left|x-x_{0}\right|<\delta_{0} r$ and $u(x) \geq L+\varepsilon$, then $x$ can be joined to $x_{0}$ by a continuum $\Gamma, \Gamma \subset \frac{3}{4} \bar{B}$, such that $u \geq L$ on $\Gamma$.

Proof: Consider the $A$-subharmonic function $v=\frac{1}{M+\varepsilon} \max (0, u+\varepsilon-$ $L)$ in $B$. Then $0 \leq v<1$ and $v\left(x_{0}\right) \geq 2 \varepsilon^{\prime}, \varepsilon^{\prime}=\frac{\varepsilon}{M+\varepsilon}>0$. By Proposition 3.1 there is a number $\delta_{0}=\delta_{0}\left(n, p, \varepsilon^{\prime}, \alpha, \beta\right)>0$ such that if $\left|x-x_{0}\right|<\delta_{0} r$ and $v(x) \geq 2 \varepsilon^{\prime}$, then $x$ and $x_{0}$ belong to the same component of $\left\{v \geq \varepsilon^{\prime}\right\} \cap \frac{3}{4} \bar{B}$. If now $u(x) \geq L+\varepsilon$ and $\left|x-x_{0}\right|<\delta_{0} r$, then $v(x) \geq 2 \varepsilon^{\prime}$ and hence $x$ and $x_{0}$ belong to the same component of $\left\{v \geq \varepsilon^{\prime}\right\} \cap \frac{3}{4} \bar{B}=\{u \geq L\} \cap \frac{3}{4} \bar{B}$. The lemma follows.

\section{TRACTS AND GROWTH}

In this section we study tracts and growth of entire $A$-subharmonic functions in $R^{n}$. Our results extend works of M. N. M. Talpur, see [8, pp. 177-185], and V. M. Miklyukov [17].

4.1. Limit components. Suppose that $u$ is $A$-subharmonic in $\Omega$. If $x_{0} \in \Omega$ and $u\left(x_{0}\right) \geq L>-\infty$, as in [8, p. 177] we define the limit component $K=K\left(x_{0}, L, \Omega\right)$ of the set $\{u \geq L\}$ to be the union of all continua which lie in $\Omega$, contain $x_{0}$, and on which $u \geq L$. Equivalently, if $\Omega_{j} \subset \subset \Omega_{j+1} \subset \subset \Omega$ is any exhaustion of $\Omega, \cup_{j} \Omega_{j}=\Omega$, then $K\left(x_{0}, L, \Omega\right)=\cup_{j} K\left(x_{0}, L, \bar{\Omega}_{j}\right)$ where $K\left(x_{0}, L, \bar{\Omega}_{j}\right)$ is the $x_{0}$-component of the compact set $\{u \geq L\} \cap \bar{\Omega}_{j}$.

4.2. Lemma. Suppose that $u$ is $A$-subharmonic in $\Omega$ and $K=K\left(x_{0}, L, \Omega\right)$ is a limit component of $\{u \geq L\}$. Set

$$
v(x)= \begin{cases}u(x), & x \in K, \\ L, & x \in \Omega \backslash K .\end{cases}
$$

Then $v$ is $A$-subharmonic in $\Omega$.

Proof: Let $\Omega_{j} \subset \subset \Omega_{j+1} \subset \subset \Omega$ be an exhaustion of $\Omega$, and let $K_{j}$ be the $x_{0}$-component of $\{u \geq L\} \cap \bar{\Omega}_{j}$. Then $K=\cup_{j} K_{j}$. By Lemma 3.2 the functions

$$
v_{j}(x)= \begin{cases}u(x), & x \in K_{j}, \\ L, & x \in \Omega_{j} \backslash K_{j},\end{cases}
$$

are $A$-subharmonic in $\Omega_{j}$. Clearly $v_{j}(x) \leq v_{j+1}(x)$ for $x \in \Omega_{j}$, and $\lim v_{j}(x)=v(x)$ for $x \in \Omega$. It thus remains to show that $v$ is u.s.c. at 
each point $x \in \Omega$ (see [11]). To do this, we argue as in [8, p. 179]: Since the claim is immediate for $x$ not in $\bar{K}$, we may assume that $x \in \bar{K}$. Suppose first $x \in K$. If $\varepsilon>0$ is given, there is a neighborhood $U$ of $x$ such that $u(y)<u(x)+\varepsilon=v(x)+\varepsilon$ for $y$ in $U$. Thus, $v(y) \leq$ $\max (u(y), L)<v(x)+\varepsilon$ for $y$ in $U$, whence $v$ is u.s.c. at $x$. Next suppose that $x \in \bar{K} \backslash K$. Then $v(x)=L$, and if $u(x) \leq L$, we obtain as above that $v$ is u.s.c. at $x$; thus suppose $u(x)>L$. Let $B=B(x, r)$ be a ball such that $2 B \subset \Omega$ and let $K_{1}$ be the $x$-component of $\{y \in \Omega: u(y) \geq$ $L\} \cap \bar{B}$. Then $K_{1} \cap K=\emptyset$, since otherwise $x$ would be in $K$. Given $\varepsilon$, $0<\varepsilon<u(x)-L$, there is by Lemma 3.11 a neighborhood $U$ of $x$ such that $u(y)<L+\varepsilon$ whenever $y$ is in $U \backslash K_{1}$. Since $v=L$ in $K_{1}$, then $v(y)<L+\varepsilon=v(x)+\varepsilon$ for $y$ in $U$, and we conclude that $v$ is u.s.c. at $x$. This completes the proof of the lemma.

A limit component $K=K\left(x_{0}, L, \Omega\right)$ of $\{u \geq L\}$ is said to be thin if $u \equiv L$ in $K$; otherwise we call $K$ thick. We next show that the number of thick limit components is limited in that there are only countably many of them; this is no longer true for thin limit components as shown by Talpur [19].

4.3. Proposition. Suppose that $u$ is $A$-subharmonic in $\Omega$ and that $K=K\left(x_{0}, L, \Omega\right)$ is a limit component of $\{u \geq L\}$. Then $\bar{K} \cap \partial \Omega \neq \emptyset$ (or $K$ is unbounded if $\Omega=\mathbb{R}^{n}$ ). If $K$ is thick, then $K$ has positive $n$-measure.

Proof: The first assertion is clear if $K$ is thick, for otherwise the function $v$ in Lemma 4.2 would violate the maximum principle. If $K$ is thin, the argument goes exactly as in [8, pp. 180-181].

To prove the second assertion, suppose that $K$ is thick. Let $v$ be the $A$-subharmonic function in Lemma 4.2. Then there is $y \in K$ such that $v(y)>L$, and by (2.8) there is a neighborhood $U$ of $y$ such that $\operatorname{essup}_{U} v>L$. It follows that $0<|\{v>L\}| \leq|K|$ as desired. (Observe that $K$ is $F_{\sigma}$, hence measurable.)

4.4. Theorem. Suppose that $u$ is $A$-subharmonic in $\mathbb{R}^{n}$ and not bounded above. Let $K$ be a limit component of $\{u \geq L\}$ such that $u \leq M<\infty$ on $K$.

(a) If $p \geq n$, then $K$ is thin.

(b) If $p<n$ and $K$ is thick, then there is at least one thick component $K_{1}$ of $\left\{u \geq L_{1}\right\}$ which is disjoint from $K$; moreover, $u$ is unbounded on each such thick component.

Proof: Suppose first that $p \geq n$ and $u$ is bounded on some thick component $K$. Then the function $v$ in Lemma 4.2 is a bounded, non-constant 
A-subharmonic function in $\mathbb{R}^{n}$. However, it follows from $[\mathbf{1 0},(2.25)]$, see also $[\mathbf{1 1}, 5.4]$, that, if $p \geq n$, such functions do not exist. This establishes (a).

The proof of (b) is more involved. We use the ideas from the proof of Proposition 3.1 .

Thus, let $p<n$ and let $K$ be a thick limit component of $\{u \geq L\}$ such that $u \leq M<\infty$ on $K$. Since $u$ is not bounded above, necessarily $u(y)>M$ for some $y \in \mathbb{R}^{n}$, and it follows that there is a thick limit component, say $K_{1}$ of $\left\{u \geq L_{1}\right\}$, which is disjoint from $K$. Set

$$
\begin{aligned}
v(x) & = \begin{cases}u(x)-L, & x \in K \\
0, & \text { otherwise }\end{cases} \\
v_{1}(x) & = \begin{cases}u(x)-L_{1}, & x \in K_{1}, \\
0, & \text { otherwise }\end{cases}
\end{aligned}
$$

The functions $v$ and $v_{1}$ are non-negative, and they are also $A$-subharmonic in $\mathbb{R}^{n}$ by Lemma 4.2 . Choose $r_{0}>0$ such that $v\left(x_{0}\right), v_{1}\left(y_{0}\right) \geq \varepsilon>0$ for some $x_{0}, y_{0} \in B\left(0, r_{0}\right)$. Since $v \leq M^{\prime}=M-L$, it follows from Lemma 3.4 that for all $r>0$

$$
\varepsilon \leq 2 M^{\prime} \exp \left(-c_{1} \int_{0}^{r} \varphi_{p}\left(x_{0}, E, s\right) \frac{d s}{s}\right)
$$

where $E=\left\{x \in \mathbb{R}^{n}: v(x)=0\right\}$. By arguing as in the proof of Proposition 3.1 we find a sequence $r=s_{0}>s_{1}>s_{2}>\ldots>0$ converging to zero such that

$$
2 s_{j+1} \leq s_{j} \leq c_{5} s_{j+1}
$$

and

$$
\theta\left(x_{0}, E, s_{j+1}\right)<c_{2}
$$

where $c_{5}=c_{5}\left(n, p, \alpha, \beta, \varepsilon, M^{\prime}\right)>0, c_{2}=c_{2}(n, p, \alpha, \beta)=2^{-1-\xi} c_{0}^{-1}>0$, and $j=0,1,2, \ldots$.

Since $\left\{v_{1}>0\right\} \subset E$, again as in the proof of Proposition 3.1 we obtain

$$
\begin{aligned}
\sup _{B\left(x_{0}, s_{j+1}\right)} v_{1} & \leq c_{0} 2^{\xi} f_{B\left(x_{0}, s_{j}\right)} v_{1} d x \\
& \leq c_{0} 2^{\xi} \frac{\left|\left\{v_{1}>0\right\} \cap B\left(x_{0}, s_{j}\right)\right|}{\left|B\left(x_{0}, s_{j}\right)\right|} \sup _{B\left(x_{0}, s_{j}\right)} v_{1} \\
& \leq c_{0} 2^{\xi} c_{2} \sup _{B\left(x_{0}, s_{j}\right)} v_{1} \leq \frac{1}{2} \sup _{B\left(x_{0}, s_{j}\right)} v_{1} \\
& \leq \ldots \leq 2^{-j} \sup _{B\left(x_{0}, r\right)} v_{1}=2^{-j} M_{1}
\end{aligned}
$$


where $M_{1}=\sup _{\mathbb{R}^{n}} v_{1}$. Now for each $j$ one can choose $r=r(j)>0$ such that $B\left(0, r_{0}\right) \subset B\left(x_{0}, s_{j+1}\right)$, whence

$$
\varepsilon \leq \sup _{B\left(x_{0}, s_{j+1}\right)} v_{1} \leq 2^{-j} M_{1}
$$

and letting $j \rightarrow \infty$ yields $M_{1}=\infty$ as required. The proof of Theorem 4.4 is complete.

4.5. CoRollary. Suppose that $u$ is A-subharmonic in $\mathbb{R}^{n}$ and not bounded above. There is a number $L_{0}$ such that if $L>L_{0}$, then $u$ is unbounded on each thick limit component of $\{u \geq L\}$.

Proof: If there is a thick limit component $K$ such that $u \leq L_{0}<\infty$ on $K$, then by Theorem $4.4 u$ is unbounded on each thick component of $\{u \geq L\}, L>L_{0}$.

4.6. Tracts. Suppose that $u$ is $A$-subharmonic in $\mathbb{R}^{n}$ and not bounded above. If $L_{0}$ is the number in Corollary 4.5 , then, for $L_{0}<L_{1}<L_{2}$, each thick limit component of $\left\{u \geq L_{1}\right\}$ contains at least one thick limit component of $\left\{u \geq L_{2}\right\}$. Therefore, the limit

$$
\lim _{L \rightarrow \infty} N(L)=N_{0}
$$

exists where $N(L)$ is the number of thick limit components of $\{u \geq L\}$. The number $N_{0}, 0 \leq N_{0} \leq \infty$, is the number of tracts of an entire $A$-subharmonic function (we define $N_{0}=0$ if and only if $u$ is bounded above). Note that if $N_{0}<\infty$, then there is $L_{1}<\infty$ such that each thick limit component of $\{u \geq L\}$ contains exactly one limit component of $\left\{u \geq L^{\prime}\right\}$ for $L_{1}<L<L^{\prime}$.

The above terminology is again adapted from [8].

We close this section by proving a result which can be viewed as a generalization of the celebrated theorem of L. V. Ahlfors, see e.g. [1], $[8$, p. 184].

The lower order $\lambda$ of an entire $A$-subharmonic function $u$ is defined as

$$
\lambda=\liminf _{r \rightarrow \infty} \frac{\log M(r)}{\log r}
$$

where $M(r)=\sup _{B(0, r)} u$.

4.7. THEOREM. There is an increasing continuous function $\lambda:[0, \infty] \rightarrow$ $[0, \infty]$ such that if $u$ is an entire $A$-subharmonic function in $\mathbb{R}^{n}$, and if $N_{0}$ is the number of tracts of $u$, then the lower order of $u$ is not less than 


\section{HEINONEN}

$\lambda\left(N_{0}\right)$. The function $\lambda$ depends only on $n, p, \alpha$, and $\beta$, and $\lambda(t) \rightarrow \infty$ as $t \rightarrow \infty$.

Proof: The proof is reminiscent of the one given in [8, pp. 174-176]. Since the submean-value property has to be replaced by the weaker inequality (2.11), the results are not as sharp as in the subharmonic case. For convenience we sketch the proof.

We may suppose that $N_{0}>2 c_{0}$ where $c_{0} \geq 1$ is the constant in (2.11). Fix an integer $l, 2 c_{0}<l \leq N_{0}$, and choose a ball $B_{0}=B\left(0, r_{0}\right)$ such that there are $l$ distinct thick limit components $K_{j}$ of $\left\{u \geq L_{j}\right\}, j=1,2, \ldots, l$, which intersect $B_{0}$ and on which $u$ is unbounded. Define the functions

$$
v_{j}(x)= \begin{cases}u(x)-L_{j}, & x \in K_{j} \\ 0, & \text { otherwise }\end{cases}
$$

then each $v_{j}$ is non-negative and $A$-subharmonic in $\mathbb{R}^{n}$. Moreover, we may assume that $M_{j}(r)=\sup _{B(0, r)} v_{j}>0$ for all $r \geq r_{0}$. Let

$$
\theta_{j}(r)=\theta\left(0,\left\{v_{j}>0\right\}, r\right)
$$

and

$$
T_{j}(r)=f_{B(0, r)} v_{j} d x
$$

For $\gamma>1$ and $k=0,1,2, \ldots$ denote $r_{k}=r_{0} \gamma^{k}$. The formula (2.11) implies for $j=1, \ldots, l$ and $k=0,1,2, \ldots$

$$
\begin{aligned}
M_{j}\left(r_{k}\right) & \leq c_{0}\left(\frac{\gamma}{\gamma-1}\right)^{\xi} T_{j}\left(r_{k+1}\right) \\
& \leq c_{0}\left(\frac{\gamma}{\gamma-1}\right)^{\xi} \theta_{j}\left(r_{k+1}\right) M_{j}\left(r_{k+1}\right)
\end{aligned}
$$

Thus

$$
\prod_{j=1}^{l} M_{j}\left(r_{k}\right) \leq\left(\frac{c_{0}}{l}\right)^{l}\left(\frac{\gamma}{\gamma-1}\right)^{l \xi} \prod_{j=1}^{l} M_{j}\left(r_{k+1}\right),
$$

where we also noticed that

$$
\left(\prod_{j=1}^{l} \theta_{j}\left(r_{k+1}\right)\right)^{1 / l} \leq \frac{1}{l} \sum_{j=1}^{l} \theta_{i}\left(r_{k+1}\right) \leq \frac{1}{l} .
$$


Since $c_{0} / l<\frac{1}{2}$, we may choose $\gamma$ so that $\frac{c_{0}}{l}\left(\frac{\gamma}{\gamma-1}\right)^{\xi}=\frac{1}{2}$; observe that $\gamma=\gamma(n, p, \alpha, \beta, l) \rightarrow 1$ as $l \rightarrow \infty$. Therefore,

$$
M_{0}\left(r_{k}\right) \leq \frac{1}{2} M_{0}\left(r_{k+1}\right)
$$

where $M_{0}\left(r_{k}\right)=\left(\prod_{j=1}^{l} M_{j}\left(r_{k}\right)\right)^{1 / l}$, and hence for any $r, \gamma^{k} r_{0} \leq r<$ $\gamma^{k+1} r_{0}$

$$
M_{0}\left(r_{0}\right) \leq 2^{-k} M_{0}(r)
$$

It follows that

$$
\frac{1}{2} M_{0}\left(r_{0}\right)\left(r / r_{0}\right)^{\rho} \leq M_{0}(r)
$$

where $\rho=\frac{\log 2}{\log \gamma} \rightarrow \infty$ as $l \rightarrow \infty$. This establishes the desired conclusion since we have $M_{0}\left(r_{0}\right)>0$.

\section{The COMPletion of The PROOF OF TheOREM 1.2}

Suppose that $u$ is $A$-subharmonic in $\mathbb{R}^{n}$ and not bounded above. We first remark that the considerations in Section 3 immediately leads to the existence of an asymptotic continuum $\Gamma$ along which $u$ tends to infinity (by an asymptotic continuum we mean a closed connected set which eventually leaves every ball $B(0, R)$ in $\left.\mathbb{R}^{n}\right)$. In particular, it is easily seen that if $u$ is continuous, then one can choose $\Gamma$ to be sectionally polygonal path. See [8, pp. 186-187] for the proofs.

It was further shown in $[8]$ that the existence of an asymptotic continuum indeed guarantees the existence of an asymptotic path once a local estimate similar to Lemma 3.11 is established. The argument in $[8, \mathrm{pp}$. 188-191] is merely topological and can be copied almost verbatim. It is left to the reader to check the details.

We conclude that Theorem 1.2 is completely established.

\section{REFERENCES}

1. L. V. Ahlfors, "Collected papers I," Birkhäuser, Boston-Basel-Stuttgart, 1982

2. L. Carleson, Asymptotic paths for subharmonic functions in $\mathbb{R}^{n}$, Ann. Acad. Sci. Fenn. Ser. A I Math. 2 (1976), 35-39

3. B. Davis and J. L. Lewis, Paths for subharmonic functions, Proc. London Math. Soc. (3) 48 (1984), 401-427.

4. B. Fuglede, Asymptotic paths for subharmonic functions, Math. Ann. 213 (1975), 261-274

5. B. Fuglede, "Asymptotic paths for subharmonic functions and polygonal connectedness of fine domains," Séminaire de Théorie du Potentiel Paris, No. 5, Lecture Notes in Mathematics 814, Springer, pp. 97-116 
6. S. Granlund, P. Lindquist and O. Martio, Conformally invariant variational integrals, Trans. Amer. Math. Soc. 277 (1983), 43-73

7. W. K. Hayman, Einige Verallgemeinerungen des Iversenschen Satzes auf subharmonische Funktionen, Jber. Deutsch. Math.-Verein. 71 (1969), 115-122

8. W. K. Hayman and P. B. Kennedy, "Subharmonic functions," Academic Press, 1976

9. J. Heinonen, Boundary accessibility and elliptic harmonic measures, to appear in Complex Variables Theory Appl

10. J. Heinonen and T. Kilpeläinen, A-superharmonic functions and supersolutions to degenerate elliptic equations, Ark. Mat 26, no 1 (1988), 87-105

11. J. Heinonen and T. Kilpeläinen, Polar sets for supersolutions of degenerate elliptic equations, to appear in Math. Scand

12. J. Heinonen and T. Kilpeläinen, On the Wiener criterion and quasilinear obstacle problems, to appear in Trans. Amer. Math. Soc

13. F. Iversen, Sur quelques propriétés des fonctions monogènes au voisinage d'un point singulier, Öfv. af Finska Vet. Soc. Forh. 58 A No. 25 (1915-1916)

14. T. Lyons, Finely holomorphic functions, J. Funct. Anal. 37 (1980), 1-18

15. O. Martio, S. Rickman and J. Väisälä, Topological and metric properties of quasiregular mappings, Ann. Acad. Sci. Fenn. Ser. A I Math. 488 (1971), 1-31

16. V. G. Maz'ya, The continuity at a boundary point of the solutions of quasilinear elliptic equations, Vestnik Leningrad. Univ. Mat. Mekh. Astronom. 25, no 13 (1970), 42-55. (Russian)

17. V. M. Miklyukov, On the asymptotic properties of subsolutions of quasilinear equations of elliptic type and mappings with bounded distortion, Mat. Sb. (N. S.) 111 (153), no 1 (1980), 42-66. (Russian)

18. Yu. G. Reshetnyak, "Space mappings with bounded distortion," Izdat. Nauka, Novosibirsk, 1982. (Russian)

19. M. N. M. Talpur, A subharmonic analogue of Iversen's theorem, Proc. London Math. Soc. (3) 31 (1975), 129-148

20. M. N. M. Talpur, On the existence of asymptotic paths for subharmonic functions in $\mathbb{R}^{n}$, Proc. London Math. Soc. 32 (1976), 181-192

Keywords. A-subharmonic functions, asymptotic paths, tracts

1980 Mathematics subject classifications: $31 \mathrm{C05}, 35 \mathrm{~J} 70$

University of Jyväskylä

Department of Mathematics

Seminaarinkatu 15

SF-40100 Jyväskylä

Finland

Universität Bonn

Bonn

FRG

Current address: University of Michigan

Department of Mathematics

Ann Arbor, MI 48109-1003

USA

(Received July 18, 1988) 